\title{
Preventive effects of Lentinus edodes on homocysteinemia in mice
}

\author{
HYUN YANG, INHO HWANG, SUN KIM, CHANGHWAN AHN, EUI-JU HONG and EUI-BAE JEUNG \\ Laboratory of Veterinary Biochemistry and Molecular Biology, College of Veterinary Medicine, \\ Chungbuk National University, Cheongju, Chungbuk 361-763, Republic of Korea
}

Received February 25, 2013; Accepted May 20, 2013

DOI: 10.3892/etm.2013.1130

\begin{abstract}
Homocysteinemia is associated with cardiovascular and neuronal degenerative diseases. Deficiencies of the B vitamins lead to high homocysteine serum levels. Lentinus edodes (L.edodes) is also known as the Shiitake mushroom and may have beneficial effects on vascular and lipid metabolic diseases, including hypertension, homocysteinemia and lipidemia. In this study, we induced a homocysteinemia-like condition in mice by the administration of a folate- and vitamin B12-deficient diet and evaluated the effect of $L$. edodes on the homocysteinemia-like condition. Homocysteinemia was induced by the administration of a diet deficient in folate and vitamin B12 (DFV) for 6 weeks to mice aged 4-10 weeks. The homocysteinemic mice were treated with $L$. edodes flour (5, 10 and $20 \%$ ), eritadenine $(10 \mathrm{mg} / \mathrm{kg}$ ) or DFV only (negative control) for 2 weeks. The DFV induced a significant increase in serum homocysteine levels. The increased homocysteine serum levels were reduced by eritadenine and L.edodes flour $(5,10$ and $20 \%$ ). Hepatic levels of S-adenosyl-L-homocysteine hydrolase (SAH) were significantly higher under DFV administration and the elevated SAH levels were reduced by treatment with L. edodes in a dose-dependent manner. The mRNA expression levels of DNA methyl transferases, DNMT1 and DNMT3a, were reduced in the DFV group, and the reduced levels of DNMT1 and DNMT3a mRNA expression were recovered in the eritadenine and L.edodes (5, 10 and 20\%) groups. These results suggest that components of $L$. edodes, including eritadenine may have beneficial effects on hyperhomocysteinemia and its therapeutic effects may be involved in the regulation of DNA methylation-related genes in mice.
\end{abstract}

\section{Introduction}

The Shiitake mushroom, or Lentinus edodes (L. edodes) has been recognized for having beneficial bioactivities. Shiitake

Correspondence to: Professor Eui-Bae Jeung, Laboratory of Veterinary Biochemistry and Molecular Biology, College of Veterinary Medicine, Chungbuk National University, Heungduk-gu, 52 Naesudongro, Cheongju, Chungbuk, 361-763, Republic of Korea E-mail: ebjeung@chungbuk.ac.kr

Key words: Lentinus edodes, hyperhomocysteinemia, folate, vitamin B12, eritadenine mushrooms are an important ingredient in Asian foods, since they possess a desirable taste and odor. Additionally, this fungus has an excellent nutritional value with high levels of vitamins B and D 1). A bioactive compounds from L.edodes, eritadenine, has been shown to exert anti-hypercholemic effects in previous studies $(2,3)$. In addition to this, eritadenine may affect lipid metabolism through the inhibition of S-adenosyl homocysteine hydrolase (SAH) (4-6).

Homocysteine is a non-protein amino acid that is biosynthesized through the metabolism of methionine (6). Elevated levels of homocysteine have been associated with number of diseases, including heart failure and bone disorders. High homocysteine concentrations increase the susceptibility to endothelial injury, which leads to inflammation in various tissues and may result in ischemic injury and metabolic imbalances (7-9). In humans, normal plasma levels of total homocysteine are $5-15 \mathrm{mmol} / \mathrm{l}$. An increase in this amino acid to $5 \mu \mathrm{mol} / 1$ is associated with an elevated risk of coronary heart disease by $60-80 \%$ for males and females (10). In hyperhomocysteinemic patients, high levels of homocysteine are significantly reduced by folic acid and vitamin B12 supplementation (11). Deficiencies in B vitamins (vitamin B6, 9 and 12) may lead to hyperhomocysteinemia (12). In addition, folate and vitamin B12 deficiencies cause DNA damage, which may result from hypomethylation $(3,13)$.

DNA methylation is an important epigenetic mechanism that selectively regulates the expression of targeted genes and is associated with various cardiac diseases (14). Methylation at the promoter region controls gene transcription and in turn attracts histone deacetylases (HDACs). DNA methylation is mediated by DNA methyl transferase (DNMT); this enzyme uses S-adenosyl methionine (SAM) as a methyl group source. DNMTs include DNMT1, 2 and 3. Homocysteine selectively reduces the activity of DNMT1, resulting in increased levels of SAH in human endothelial cells (15).

The nutritional role of dietary L. edodes has not yet been elucidated in the mouse model of hyperhomocysteinemia. In the present study, we investigated the effect of L.edodes in a mouse model of hyperhomocysteinemia induced by a folateand vitamin B12-deficient diet (DFV) during the growth stage of the animals (between 4 and 12 weeks of age). The serum and hepatic levels of homocysteine were measured using serum chemistry and high performance liquid chromatography (HPLC). Expression of DNMTs in the liver was also evaluated in order to determine the potential anti-hyperhomocysteinemic effects of L.edodes. 


\section{Materials and methods}

Experimental animals. ICR mice (4 weeks-old) were obtained from Koatech (Pyeongtaek, Gyeonggi, South Korea). All animals were housed in polycarbonate cages and acclimated in an environmentally controlled room (temperature, $23 \pm 2^{\circ} \mathrm{C}$; relative humidity, $50 \pm 10 \%$; frequent ventilation; and a 12/12-h light-dark cycle) prior to use. The mice $(n=60)$ were divided into six groups ( $\mathrm{n}=10$ per group). Homocysteinemia was induced in five groups by the administration of DFV for 6 weeks.

To assess the preventative effect of L. edodes on hyperhomocysteinemia, mice received DFV alone as a negative control (NC), DFV + eritadenine $(10 \mathrm{mg} / \mathrm{kg})$ as a positive control (PC) or DFV + 5\% (T1; w/w), $10 \%(\mathrm{~T} 2 ; \mathrm{w} / \mathrm{w})$ or $20 \%(\mathrm{~T} 3 ; \mathrm{w} / \mathrm{w})$ L.edodes, from 10- to 12 weeks of age. A sham group received AIN-93M pellets. The body weights of the mice were measured before and after the experiment. The Ethics Committee of Chungbuk National University (Cheongju, Korea) approved all experimental procedures.

Measurement of SAM, SAH and serum homocysteine levels. Frozen mouse liver samples were homogenized with $0.4 \mathrm{M}$ $\mathrm{HClO}_{4}$ buffer. The tissue homogenates were centrifuged at $2,000 \mathrm{x} \mathrm{g}$ at $4^{\circ} \mathrm{C}$ for $20 \mathrm{~min}$. Supernatants were filtered with a $0.45-\mu \mathrm{m}$ filter. SAM and SAH concentrations were measured with Shimadzu LC-10 HPLC apparatus (Tokyo, Japan) equipped with a $250 \times 4.6-\mathrm{mm}$ Ultrasphere 5- $\mu \mathrm{m}$ ODS Betasil analytical column (Thermo Hypersil-Keystone, Runcorn, UK) according to a previously described protocol (16). Blood was collected from each mouse, transferred to serum separator tubes and centrifuged at $400 \mathrm{x} \mathrm{g}$ for $20 \mathrm{~min}$. The supernatant was transferred into $1.5 \mathrm{ml}$ tubes and analysis of serum homocysteine levels was performed using a chemiluminescent immunoassay method with an ADVIA Centaur Assay system (Siemens Medical Solution Diagnostics, Dublin, Ireland) according to the manufacturer's instructions.

RNA extraction and quantitative PCR. Total RNA was extracted from mouse liver using TRIzol reagent (Invitrogen Life Technologies, Carlsbad, CA, USA) according to the manufacturer's instructions. The RNA concentration was determined using an Epoch micro-plate spectrophotometer (BioTek Instruments, Inc., Winooski, VT, USA) at an absorbance of $260 \mathrm{~nm}$ and RNA quality was evaluated by electrophoresis in $1 \%$ agarose gels. Total RNA ( $1 \mathrm{mg}$ ) was reverse transcribed into first-strand complementary DNA (cDNA) using Moloney murine leukemia virus reverse transcriptase (Invitrogen Life Technologies) and a random primer (9-mer; Takara Bio Inc., Shiga, Japan). Each cDNA sample (1 ml) was amplified by $10 \mathrm{ml} 2 \mathrm{X} \mathrm{SYBR}^{\circledR}$ Premix Ex Taq $^{\mathrm{TM}}$ (Takara Bio Inc.) and $10 \mathrm{pmol}$ each primer. Amplification was performed in a 7300 Real time PCR System (Applied Biosystems, Foster City, CA, USA) using the following parameters: denaturation at $95^{\circ} \mathrm{C}$ for $5 \mathrm{~min}$, then 40 cycles of denaturation at $95^{\circ} \mathrm{C}$ for $30 \mathrm{sec}$, annealing at $60^{\circ} \mathrm{C}$ for $30 \mathrm{sec}$ and extension at $72^{\circ} \mathrm{C}$ for $45 \mathrm{sec}$. The oligonucleotide primer sequences used in this study were as follows: 5'-AAC CAA GCA AGA AGT GAA GCC C-3' (sense) and 5'-GCA AAA TGA GAT GTG ATG GTG G-3' (antisense) for DNMT1 (product size, 185 bp); 5'-GGA GGA
Table I. Body weight and daily intake.

\begin{tabular}{|c|c|c|c|}
\hline \multirow[b]{2}{*}{ Group } & \multicolumn{2}{|c|}{ Weight (g) } & \multirow[b]{2}{*}{$\begin{array}{c}\text { Daily } \\
\text { intake }(\mathrm{g})\end{array}$} \\
\hline & Baseline & $\begin{array}{c}8 \text { weeks } \\
\text { later }\end{array}$ & \\
\hline Sham & $37.1 \pm 0.9$ & $40.1 \pm 0.3$ & $4.2 \pm 0.5$ \\
\hline Negative & $37.2 \pm 0.9$ & $41.3 \pm 3.4$ & $4.6 \pm 0.9$ \\
\hline Positive & $37.6 \pm 0.7$ & $41.0 \pm 0.5$ & $4.6 \pm 0.6$ \\
\hline $5 \%$ L. edodes & $37.5 \pm 0.9$ & $41.9 \pm 0.7$ & $4.2 \pm 0.5$ \\
\hline $10 \%$ L. edodes & $37.8 \pm 0.1$ & $39.7 \pm 1.3$ & $4.4 \pm 0.8$ \\
\hline $20 \%$ L.edodes & $37.6 \pm 0.8$ & $39.1 \pm 2.1$ & $4.6 \pm 1.0$ \\
\hline
\end{tabular}

ATG TGC CAA AAC TG-3' (sense) and 5'-GCA GTT GTT GTT TCC GCA C-3' (antisense) to amplify DNMT3a (product size, $132 \mathrm{bp}$ ); and 5'-AAC AGC ATC GGC AGG AAC-3' (sense) and 5'-ATC TTT CCC CAC ACG AGG-3' (antisense) for DNMT3b (product size, $249 \mathrm{bp}$ ). The oligonucleotide primer sequences used to amplify $\beta$-actin (product size, 131 bp) were: 5'-GGC ACC CAG CAC AAT GAA G-3' (sense) and 5'-GCA AAA TGA GAT TGT ATG GTG G-3' (antisense). The relative expression levels of DNMT1 (normalized to the level of $\beta$-actin) in each sample were determined using RQ software (Applied Biosystems). All real-time PCR experiments were repeated twice.

Data analysis. Data are presented as the mean \pm standard error of the mean (SEM) and were analyzed with a one-way analysis of variance (ANOVA) followed by Tukey's multiple comparison test. Statistical analyses were performed using Prism Graph Pad (v.4.0; GraphPad Software Inc., San Diego, CA, USA). $\mathrm{P}<0.05$ was considered to indicate a statistically significant difference.

\section{Results}

Body weight and daily dietary intake. Differences in bodyweight and daily intake between mice who ate a normal diet and those who received DFV for 6 weeks were evaluated. The mice from the six groups had similar body weight gains as shown in Table I. The body weights of mice in the sham, NC and PC (eritadenine) groups did not change in response to the DFV. The groups treated with L.edodes flour (10 and 20\%) had lower final body weights compared with those of the other groups at 6 weeks; however, the differences in weight were not significant.

Serum levels of homocysteine in hyperhomocysteinemic mice. Dietary supplementation with L. edodes affected the serum levels of homocysteineine in mice with hyperhomocysteinemia, caused by the DFV (Fig. 1). The DFV resulted in a marked increase in homocysteine levels from $2.9 \pm 0.1$ to $7.3 \pm 0.8 \mathrm{mmol} / \mathrm{l}$ after 6 weeks. The addition of dietary eritadenine $(10 \mathrm{mg} / \mathrm{kg})$ or L. edodes flour $(5,10$ or $20 \%)$ attenuated the rise in serum homocysteine levels caused by the DFV. In the groups receiving L.edodes (5, 10 and 20\%), the levels of homocysteine were reduced in a dose-dependent manner compared with those in the $\mathrm{NC}$ group. 
Table II. Hepatic SAH and SAM levels, and SAM/SAH ratios in hyperhomocysteinemic mice.

\begin{tabular}{|c|c|c|c|}
\hline Group & $\begin{array}{c}\mathrm{SAH} \\
\text { (nmol/g tissue) }\end{array}$ & $\begin{array}{c}\text { SAM } \\
\text { (nmol/g tissue) }\end{array}$ & $\begin{array}{l}\mathrm{SAM} / \mathrm{SAH} \\
\text { ratio }\end{array}$ \\
\hline Sham & $5.2 \pm 0.2$ & $10.8 \pm 0.6$ & $2.1 \pm 0.1$ \\
\hline Negative & $13.5 \pm 0.5$ & $7.7 \pm 0.4$ & $0.58 \pm 0.04$ \\
\hline Positive & $6.6 \pm 0.4^{\mathrm{a}}$ & $10.3 \pm 0.9^{\mathrm{a}}$ & $1.59 \pm 0.19^{\mathrm{a}}$ \\
\hline $5 \%$ L.edodes & $8.7 \pm 0.4^{a}$ & $7.8 \pm 0.8$ & $0.90 \pm 0.08^{\mathrm{a}}$ \\
\hline $10 \%$ L. edodes & $7.5 \pm 0.4^{\mathrm{ab}}$ & $8.0 \pm 0.8$ & $1.01 \pm 0.07^{\mathrm{ab}}$ \\
\hline $20 \%$ L.edodes & $6.5 \pm 0.3^{\mathrm{ac}}$ & $8.2 \pm 1.0$ & $1.16 \pm 0.08^{\mathrm{ac}}$ \\
\hline
\end{tabular}

${ }^{\mathrm{a}} \mathrm{P}<0.05$ vs. negative control; ${ }^{\mathrm{b}} \mathrm{P}<0.05$ vs. $5 \%$ L. edodes; ${ }^{\mathrm{c}} \mathrm{P}<0.05$ vs. $10 \%$ L. edodes. SAH, S-adenosyl homocysteine hydrolase; SAM, S-adenosyl methionine.

Hepatic SAH and SAM levels, and SAM/SAH ratios in hyperhomocysteinemic mice. HPLC was used to measure SAM and SAH levels, and the SAM/SAH ratios in liver tissues were calculated. Hepatic SAH levels were increased by the DFV as shown in Table II. The level of SAH was increased in the NC group. The increase in SAH levels was significantly reduced by eritadenine, as well as by L.edodes (5, 10 and 20\%) in a dose-dependent manner. In addition, the SAM/SAH ratio was reduced by the DFV and increased by dietary supplementation with eritadenine and L.edodes flour (5, 10 and 20\%).

Expression of DNMT (1 and 3a) mRNA in the livers of hyperhomocysteinemic mice. To measure the expression of hepatic DNMT1, the mice were separated into six groups (sham, NC, PC, T1, T2, and T3). As shown in Fig. 2, the level of DNMT1 mRNA was reduced by the DFV in the NC group compared with that in the sham group, and was significantly increased by eritadenine supplementation in the PC group. Hepatic DNMT1 mRNA expression was also dose-dependently increased by supplementation with L.edodes $(5,10$ and 20\%). Hepatic DNMT3b mRNA was not abundant in the liver of this mouse model while DNMT3a expression was reduced by DFV compared with the level in the sham group. This inhibitory effect was eliminated by eritadenine and all of doses of L. edodes flour (5, 10 and 20\%).

\section{Discussion}

L. edodes contains considerable concentrations of vitamins (D, B6, B9 and B12) and other beneficial compounds, including eritadenine and dietary fiber (1). This fungus is considered to be useful for treating hypercholesterolemia, inflammation, hypertension and osteoporosis (17-19). In a previous study, we examined the anti-osteoporotic effects of L.edodes and the ability of this fungus to induce the expression of duodenal and renal calcium transport channels in mice with osteoporosis-like symptoms (19). In the present study, we investigated the anti-hyperhomocysteinemic effects of $L$. edodes and determined whether it prevents increases in homocysteine levels in mice with dietary folate and vitamin B12 deficiencies. After the mice had received specialized diets for 6 weeks, starting

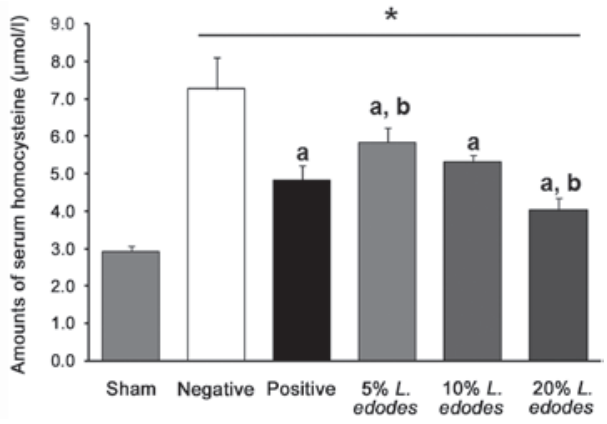

Figure 1. Serum levels of homocysteine with folate- and vitamin B12 deficiency (DFV)-induced hyperhomocysteinemia in mice. The serum homocysteine levels were measured in the six groups: AIN-93M diet only(sham), DFV (negative control), DFV + eritadenine (positive control), $\mathrm{DFV}+5 \%$ Lentinus edodes (T1), DFV $+10 \%$ Lentinus edodes $(\mathrm{T} 2)$ and DFV $+20 \%$ Lentinus edodes (T3). The level of homocysteine was examined by a chemiluminescent immunoassay method (CLIA). "P $<0.05$ vs. the sham group; ${ }^{\mathrm{a}} \mathrm{P}<0.05$ vs. the negative control; ${ }^{\mathrm{b}} \mathrm{P}<0.05$ vs. the positive control.

A
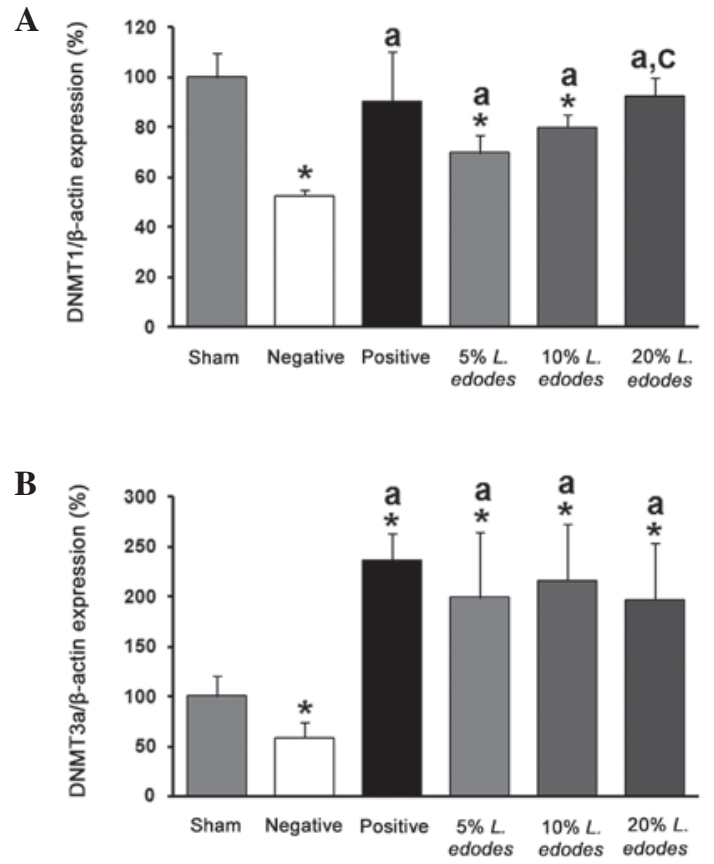

Figure 2. Pattern of DNMT1 and DNMT3a mRNA expression in mice with folate and vitamin B12 deficiency (DFV)-induced hyperhomocysteinemia. Quantitative PCR was performed to detect DNMT1 and DNMT3a mRNA in the livers of mice. Liver tissues were divided into six groups: AIN-93M diet only (sham), DFV (negative control), DFV + eritadenine (positive control), $\mathrm{DFV}+5 \%$ Lentinus edodes (T1), FVD $+10 \%$ Lentinus edodes (T2) and DFV $+20 \%$ Lentinus edodes (T3). ${ }^{*} \mathrm{P}<0.05$ vs. the sham group; ${ }^{\mathrm{a}} \mathrm{P}<0.05$ vs. the negative control; ${ }^{\mathrm{C}} \mathrm{P}<0.05$ vs. $5 \%$ L. edodes. DNMT, DNA methyl transferase.

when the animals were 6 weeks old, we observed that dietary L. edodes supplementation reduced the serum and hepatic levels of homocysteine in the hyperhomocysteinemic mice. Eritadenine was also shown to have a similar effect.

Dietary supplementation with eritadenine has been reported to effectively suppress guanidine acetic acid (GAA)-induced hyperhomocystenemia in rats (6). However, the mechanism underlying this effect has not been fully elucidated. Analyses of eritadenine content in L.edodes identified that the concentration of this compound ranged between 3.2 and $6.3 \mathrm{mg} / \mathrm{g}$ 
dried mushroom (20). The total amount of aministered eritadenine in the PC group $(10 \mathrm{mg} / \mathrm{kg})$ was lower compared with the amount contained in the dietary L. edodes $(>25 \mathrm{mg} / \mathrm{kg})$; however, the anti-hyperhomocysteinemic effects were similar.

The expression of DNMT1 mRNA, which the DFV downregulated, was increased by eritadenine and dose-dependently increased by L.edodes $(5,10$ and 20\%) in the mice with hyperhomocysteinemia compared with the level in the NC group. Similarly, the level of DNMT3a mRNA was decreased by the DFV; this inhibitory effect was abolished and the level of DNMT3a was elevated by eritadenine and all doses L. edodes (5, 10 and 20\%) compared with the sham group. A previous study demonstrated that homocysteine selectively reduces the activity of DNMT1 and increases SAH levels in human endothelial cells, but does not affect DNMT3 (15). In another study, DNMT1 and DNMT3 expression was reported to be elevated by homocysteine in human monocytes (21). The levels of DNMT1 and DNMT3 have been demonstrated to be mediated or selectively regulated by homocysteine $(15,21)$. DNMT3 levels have been found to differ between mouse liver, HUVECs and human monocytes, and may vary according to tissue type and species $(15,21,22)$. These results indicate that homocysteine is affected by upregulation of DNA methylation through the suppression of HDAC activity and increased DNMT1 and DNMT3 activities.

In conclusion, the present study demonstrated that supplementation with eritadenine and L.edodes (5, 10 and $20 \%$ ) significantly inhibited the effects of DFV-induced hyperhomocysteinemia in mice. Reduced serum and hepatic homocysteine levels illustrated the beneficial effects of L. edodes on hyperhomocysteinemia-like symptoms. Serum and hepatic homocysteine levels were significantly reduced by the administration of eritadenine and L.edodes to hyperhomocysteinemic mice with dietary folate and vitamin B12 deficiencies compared with their levels in the NC group. In addition, we examined the expression of DNMT1 and DNMT3a in the livers of mice and demonstrated that hepatic DNMT1 and DNMT3a levels were increased to the levels observed in sham animals by eritadenine and L.edodes. Based on our findings, we propose that L. edodes may improve hyperhomocysteinemic symptoms due to the beneficial compounds it contains.

\section{Acknowledgements}

This study was supported by a National Research Foundation of Korea (NRF) grant funded by the Korean Ministry of Education, Science and Technology (MEST; No. 2010-0011433).

\section{References}

1. Wasser SP: Shiitake (Lentinus edodes). In: Encyclopedia of Dietary Supplements. Coates PM, Blackman MR, Cragg GM, et al (eds). 1st edition. Marcel Dekker, New York, NY, pp653-664, 2005 .
2. Ngai PH and Ng TB: Lentin, a novel and potent antifungal protein from shitake mushroom with inhibitory effects on activity of human immunodeficiency virus-1 reverse transcriptase and proliferation of leukemia cells. Life Sci 73: 3363-3374, 2003.

3. Shimada Y, Morita T and Sugiyama K: Eritadenine-induced alterations of plasma lipoprotein lipid concentrations and phosphatidylcholine molecular species profile in rats fed cholesterol-free and cholesterol-enriched diets. Biosci Biotechnol Biochem 67: 996-1006, 2003

4. Sugiyama K, Akachi T and Yamakawa A: Hypocholesterolemic action of eritadenine is mediated by a modification of hepatic phospholipid metabolism in rats. J Nutr 125: 2134-2144, 1995.

5. Shimada Y, Morita T and Sugiyama K: Dietary eritadenine and ethanolamine depress fatty acid desaturase activities by increasing liver microsomal phosphatidylethanolamine in rats. J Nutr 133: 758-765, 2003.

6. Fukada S, Setoue M, Morita T and Sugiyama K: Dietary eritadenine suppresses guanidinoacetic acid-induced hyperhomocysteinemia in rats. J Nutr 136: 2797-2802, 2006.

7. Akalin A, Alatas O and Colak O: Relation of plasma homocysteine levels to atherosclerotic vascular disease and inflammation markers in type 2 diabetic patients. Eur J Endocrinol 158: 47-52, 2008.

8. Goldstein LB: Novel risk factors for stroke: homocysteine, inflammation, and infection. Curr Atheroscler Rep 2: 110-114, 2000.

9. Kelly PJ, Kistler JP, Shih VE, et al: Inflammation, homocysteine, and vitamin B6 status after ischemic stroke. Stroke 35: 12-15, 2004.

10. Selhub J: Homocysteine metabolism. Annu Rev Nutr 19: 217-246, 1999.

11. McCaddon A, Hudson P, Ellis D, Hill D and Lloyd A: Effect of supplementation with folic-acid on relation between plasma homocysteine, folate, and vitamin B12. Lancet 360: 173, 2002.

12. Miller JW, Nadeau MR, Smith D and Selhub J: Vitamin B-6 deficiency vs folate deficiency: comparison of responses to methionine loading in rats. Am J Clin Nutr 59: 1033-1039, 1994.

13. Kruman, II, Culmsee C, Chan SL, et al: Homocysteine elicits a DNA damage response in neurons that promotes apoptosis and hypersensitivity to excitotoxicity. J Neurosci 20: 6920-6926, 2000.

14. Hiltunen MO and Ylä-Herttuala S: DNA methylation, smooth muscle cells, and atherogenesis. Arterioscler Thromb Vasc Biol 23: 1750-1753, 2003

15. Jamaluddin MD, Chen I, Yang F, et al: Homocysteine inhibits endothelial cell growth via DNA hypomethylation of the cyclin A gene. Blood 110: 3648-3655, 2007.

16. Wagner J, Claverie $\mathrm{N}$ and Danzin C: A rapid high-performance liquid chromatographic procedure for the simultaneous determination of methionine, ethionine, S-adenosylmethionine, $\mathrm{S}$-adenosylethionine, and the natural polyamines in rat tissues. Anal Biochem 140: 108-116, 1984.

17. Kabir Y, Yamaguchi M and Kimura S: Effect of shiitake (Lentinus edodes) and maitake (Grifola frondosa) mushrooms on blood pressure and plasma lipids of spontaneously hypertensive rats. J Nutr Sci Vitaminol (Tokyo) 33: 341-346, 1987.

18. Carbonero ER, Gracher AHP, Komura DL, Marcon R, Freitas CS, Baggio $\mathrm{CH}$, Santos ARS, Torri G, Gorin PAJ and Iacomini M: Lentinus edodes heterogalactan: antinociceptive and antiinflammatory effects. Food Chem 111: 531-537, 2008.

19. Lee GS, Byun HS, Yoon KH, Lee JS, Choi KC and Jeung EB: Dietary calcium and vitamin D2 supplementation with enhanced Lentinula edodes improves osteoporosis-like symptoms and induces duodenal and renal active calcium transport gene expression in mice. Eur J Nutr 48: 75-83, 2009.

20. Enman J, Rova U and Berglund KA: Quantification of the bioactive compound eritadenine in selected strains of shiitake mushroom (Lentinus edodes). J Agric Food Chem 55: 1177-1180, 2007.

21. Jiang Y, Jiang J, Xiong J, et al: Homocysteine-induced extracellular superoxide dismutase and its epigenetic mechanisms in monocytes. J Exp Biol 211: 911-920, 2008.

22. Vizzardi E, Bonadei I, Zanini G, et al: Homocysteine and heart failure: an overview. Recent Pat Cardiovasc Drug Discov 4: 15-21, 2009. 